\title{
Noise Exposure on Human Cochlea during Cochleostomy Formation Using Conventional and A Hand Guided Robotic Drill
}

1 Abstract:

2 Objective: To investigate the disturbance induced in the cochlea during cochleostomy using conventional drill 3 and a hand guided robotic drill.

4 Study Design: The study is based on experimental measurements using the Laser Doppler Vibrometer during the drilling processes converted to Sound Pressure Levels (SPL) for comparison.

Setting: The study is based on experimental results of three sets of cochleostomies on human cadaver heads. Main Outcome Measure(s): Robotic drilling, in comparison to the conventional drilling method, creates a consistently lower level of disturbance in cochlea across the hearing frequency range.

Results: Robotic drilling, in comparison to the conventional drilling method, creates a consistently lower level of disturbance in cochlea across the hearing frequency range.

Conclusions: It is reasonable to conclude that robotic drilling has a lower possibility of creating acoustic trauma in cochlea that endangers the residual hearing of patients.

Key words - Hand guided robotic drill, cochleostomy, drill disturbances, hearing preservation

\section{INTRODUCTION}

Preserving residual hearing for patients with hearing impairments is a critical objective in ear surgery. This cautionary approach applies to cochlear implant (CI) surgery, especially for patients who still have substantial hearing at low frequencies where acoustic signals can be perceived authentically by hair cells at the apical part of the cochlea. The enhanced hearing performance following cochlear implantation indicates potential expansion of the patient group able to benefit people who have residual hearing [1].

Apart from efforts to refine electrode characteristics [1], attention has been focused on the surgical procedure, more specifically exposure to acoustic and mechanical trauma during CI surgery. Among the steps of cochlear implantation, drilling is a significant contributor to trauma caused by both the potential high level of disturbance induced and the relatively long period of surgery. A normal CI surgery takes approximately 2 hours [2]. The average period of drilling directly on the cochlea to prepare the cochleostomy is 8 minutes [3]. Cochlea can be exposed to an average sound pressure level of $89.9 \mathrm{~dB}$ SPL, maximal $118 \mathrm{~dB}$ SPL during the approximately 8minute continuous drilling period [3]. According to information provided by The American Hearing Research Foundation, persistent sound vibration louder than $85 \mathrm{~dB}$ SPL can cause permanent hearing loss [4]. The hearing mechanism of the ear cannot tolerate sound levels greater than 140dB SPL and the maximum duration the ear can be exposed to a $115 \mathrm{~dB}$ sound without permanent hearing loss is 15 minutes. When measured on temporal bones, the noise level during cochleostomy was found to be from 116 to $131 \mathrm{~dB}$ SPL, and exceeded 130dB SPL when the endosteal membrane was touched by the burr $[5,6]$.

A hand-guided robotic drill has been developed by the authors for cochlear implantation focusing on the formation of cochleostomies. Although there is an ongoing debate about the optimal procedure for opening cochlea through cochleostomy or round window (RW), sometimes cochleostomies cannot be avoided, particularly if the RW is difficult to access. Cochleostomy is considered crucial to hearing preservation, not only because it exposes the cochlea to perilymph and the risk of drill bit entering scalar vestibule, but also because the action of drilling can cause inner ear trauma. Drill induced mechanical trauma is proven to be severe in middle ear surgery, especially if the ossicular chain is drilled unintentionally. Using a robotic device to perform cochleostomy can help to improve the consistency and accuracy. An innovative tactile method to automatically discriminate mediums and structures ahead on a cutting tool trajectory has been demonstrated successfully in surgery to produce precise cochleostomies [7]. The method enables preservation of fine tissue structures by simultaneously determining the state of the process and automatically stopping the drilling if undesired drilling medium is detected. Most important, this is used to achieve high tissue preservation and low tissue trauma in surgery [8-10]. during cochleostomy formation. The tests were performed on two human cadaver heads. Three sets of cochleostomies were created. The Middle Ear Transfer Function (METF) was measured on each of the tested ear 
specimen. Drilling disturbances measured by the Laser Doppler Vibrometer (LDV) during the drilling process

\section{Method AND MATERIAL}

\section{A. Hand guided robotic drill}

Robotic approach for surgery has made its mark as a precise means of tool deployment in surgical procedures [11]. It has demonstrated consistently positive results [12-14] for certain procedures, such as laparoscopic surgery, with reduced length of stay and blood loss $[15,16]$. Several robotic devices have been developed for minimally invasive cochlear implantation [17-19]. Such robotic devices require high resolution CT images for the operator to pre-plan the drilling path $[17,18]$ or calibrate the robot [19]. During the surgery, an image navigation system is used to track the movement of the robotic arm relative to the patient. Primarily for such robotic device development is focused on creating an access tunnel to cochlea, avoiding the facial nerve during the drilling process. Similar to many other procedures, the upfront cost, surgeon training overhead, consumable and maintenance costs of an extensive system cannot be justified [20]. Meanwhile, a number of hand-guided robotic systems which are smaller and intuitive to use have been developed. For example, to assist gripping tissues (Laparoscopy), guiding hand-held instruments and in cutting applications (knee joint replacement surgery) [2024]. Hand-held robots have the advantage of being compact and easily integrated into routine surgical practice. These devices have a physically smaller footprint and make use of the available surgeon dexterity. They are typically lower in cost, require minimal setup time, and lower training overhead [25].

The concept of a hand guided robotic drill has been inspired by an automated, mechanical arm supported, robotic drill recently applied in clinical practice to produce cochleostomies [10]. The smart sensing algorithm uses information derived from coupled force and torque transient discriminating tissue boundaries/ structures ahead on the drilling path. A hand-held drill is more convenient to use than a device constrained by a mechanical support arm. From the perspective of surgeons, who are used to deploying tools by hand, it is likely to appear more intuitive to use. Previous research has proved that the flexibility in the drilling trajectory will help the control of drilling into the basal turn of the cochlea. Initial cutting without slip is achieved more readily when the drilling trajectory is perpendicular to the surface [26-27].
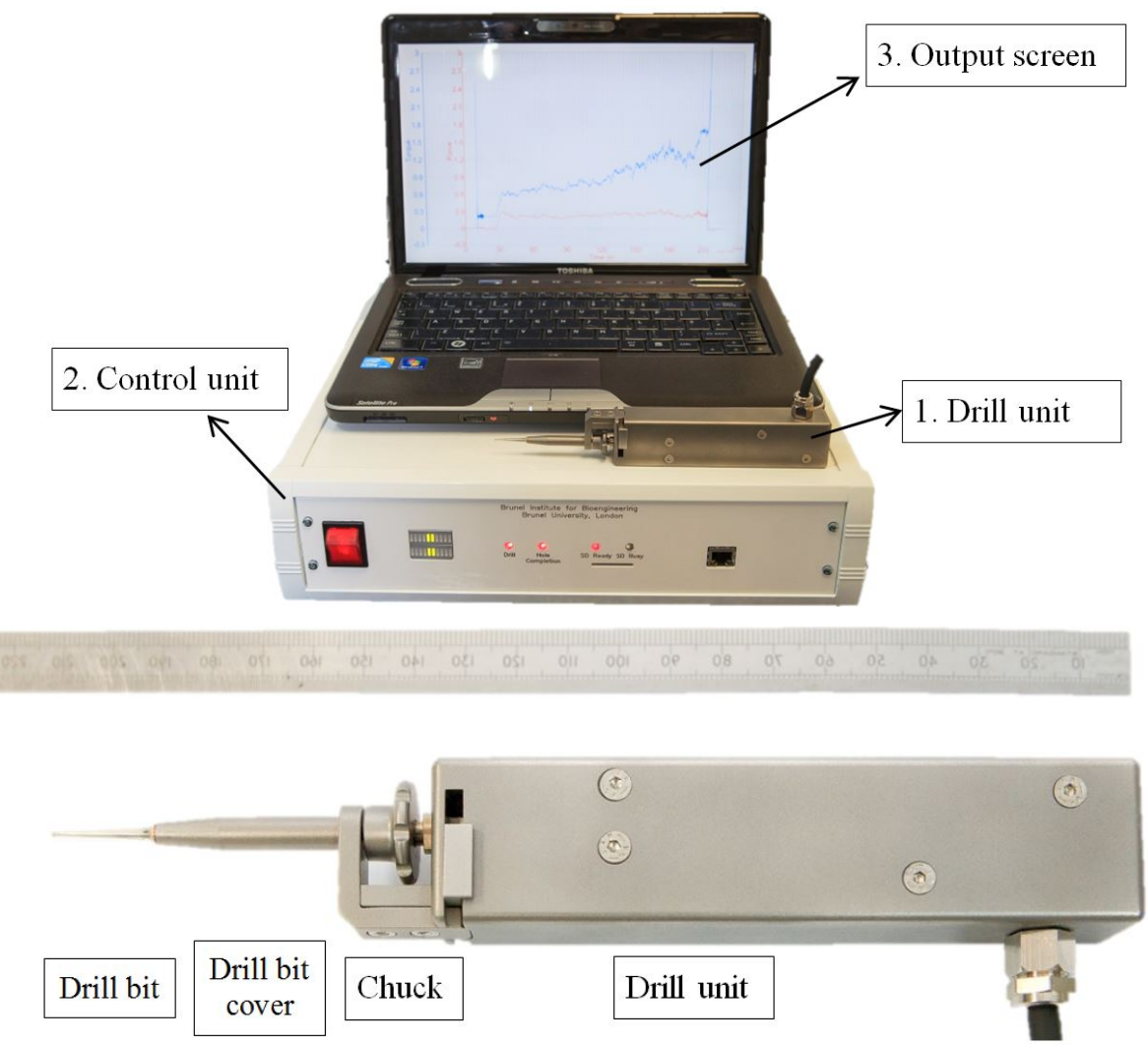

Figure 1 The experimental hand guided surgical robot drill system [27] 
The hand-guided drilling system contains three units, a drill unit, a hard-wired control unit, and an output screen. Figure 1 shows the system containing all the three units. The drill unit uses standard drill bit driven by a servo motor. The design of the chuck helps to change the drill bit easily and transfer the pushing force to the sensor inside the unit. The hard-wired control unit contains two micro-controllers. One is to provide servo control of the drill unit, and the other is to control the information communication to the output screen through ethernet. There are also LED bars on the control unit showing the pushing force during drilling. It is important to maintain the pushing force in the range between $0.5 \mathrm{~N}$ to $1.5 \mathrm{~N}$ shown as green area on the LED bars. If pushing too hard or too light, the LED bar will display red. On the output screen, a user interface is displayed to show information such as pushing force, rotation torque, and rotation speed. The system has been tested on a variety of phantoms such as raw eggs and porcine cochlear [27]. The feasibility results demonstrated the consistency and robustness when drilling on variety phantoms.

\section{B. Measurement of Round Window Velocity during Drilling Procedure}

In the context of inner ear noise measurement, there are two general approaches: the laser approach [28-29] and the microphone approach $[5,30]$. Due to the limited surface area on the cochlea - about the size of a pea with approximately $2.5 \mathrm{~mm}^{2}$ membranous exposure at round window, it has been particularly challenging to accurately observe and quantify the vibration on or within the cochlea before immense advances in microelectronics and applications of laser became ubiquitous. The laser approach measures the vibrating velocity in $\mathrm{mm} / \mathrm{s}$ at RW. The value measured is then calibrated against a reference measurement. The reference measurement is conducted by measuring the vibration amplitude in $\mathrm{mm} / \mathrm{s}$ at the $\mathrm{RW}$ when the specimen is exposed to a stimulus of a known acoustic noise level in $\mathrm{dB}$ SPL. This method enables highly accurate and non-intrusive vibrational measurement and it provides a straightforward quantification of the drilling-evoked noise levels purveyed inside the cochlea.

100 An illustration of the calibration experimental setup is provided below in Figure 2. A probe microphone ER-7C 101 (Etymotic Research, Elk Grove Village, IL 60007, USA) and a wide band earphone ER-2 (Etymotic Research), 102 both coupled to an ER1-14A disposable foam eartip (Etymotic Research), were inserted into the external ear canal.

103 The end of the probe tube of the microphone was placed at $2 \mathrm{~mm}$ lateral to the tympanic membrane. The earphone 104 was driven by a frequency logarithmic sweep signal from 0.1 to $10 \mathrm{kHz}$ at $1 \mathrm{Vrms}$ from R\&S UPV Audio Analyser (Rohde \& Schwarz, 6821 Benjamin Franklin Drive, Columbia, MD 21046, USA). According to sensitivity of the ER-2 earphone, tones delivered were at $100 \mathrm{~dB}$ SPL. A standard calibration process of the probe microphone was 107 implemented before measurement and a sensitivity value was checked against the range of 40-60 mV/Pa.

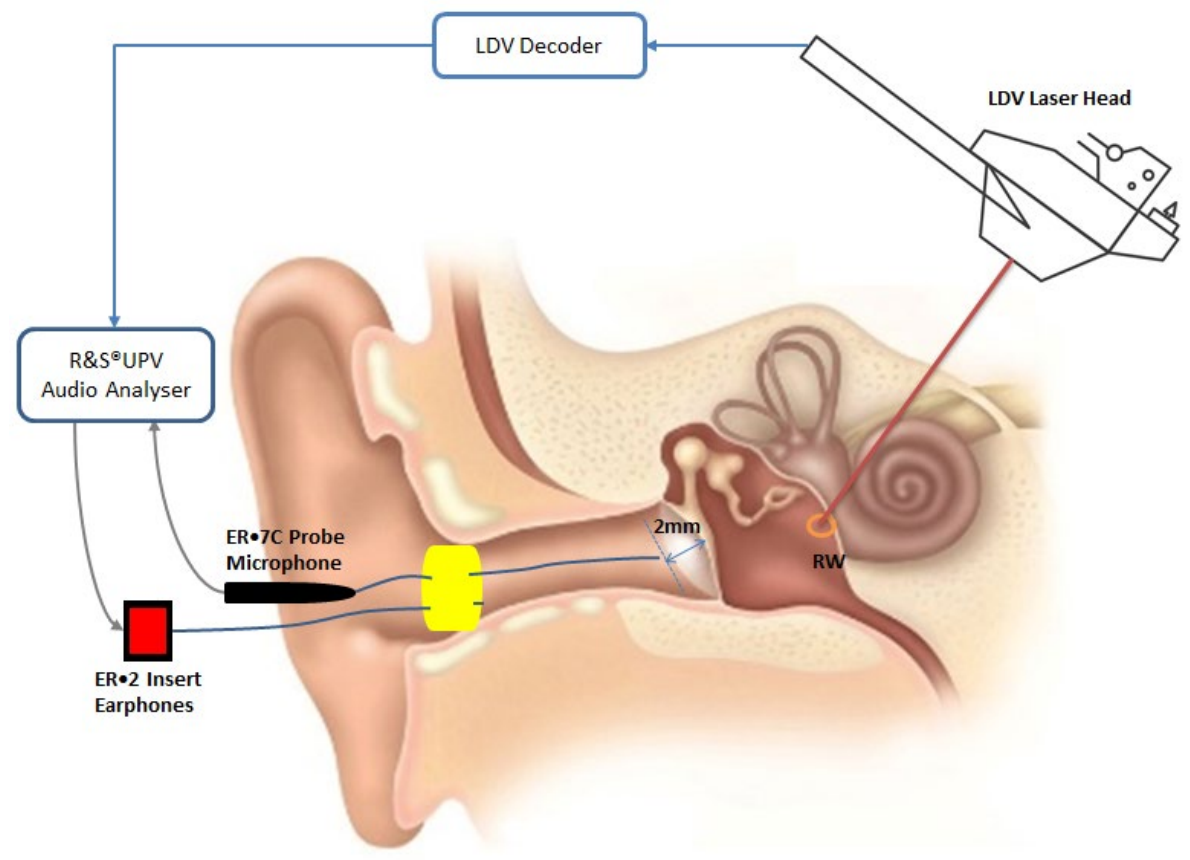

Figure 2 Schematic illustration of the calibration setup 
An LDV system was used to measure both stapes and RW velocity. The laser head part of the compact sensor head system OFV-534 (Polytec, D-76337 Waldbronn, Germany) and micro-manipulator A-HLV-MM30 (Polytec) was mounted over the lens of a surgical microscope (Wild Heerbrugg, CH - 9056 Gais, Switzerland). Self-

113

114

115

116

117

118

119

120

121

122 adhesive retroreflective tape $(<1 \mathrm{~mm} 2)$ was placed on the posterior crus of the stapes, and later at the centre of $\mathrm{RW}$, to achieve a reasonably strong reflected signal and a signal to noise ratio within the acceptable range $(>10 \mathrm{~dB})$. The reflected signal was captured and decoded by the OFV-5000 vibrometer controller (Polytec) to produce an output voltage proportional to the velocity detected. The voltage signal is fed into R\&S UPV Audio Analyser for real-time monitoring and recording. The angle of the laser to vibration axis in both cases was kept less than $45^{\circ}$ and compensated for in data analysis. After the calibration process, the hand-guided robotic drill was used to create a cochleostomy, followed by another cochleostomy on the same ear $(<1 \mathrm{~mm}$ apart $)$ with a conventional surgical drill. After each cochleostomy was made, METF was measured and checked to make sure that no significant change has incurred in the dynamics of the cochlea and the wider hearing conducting system.

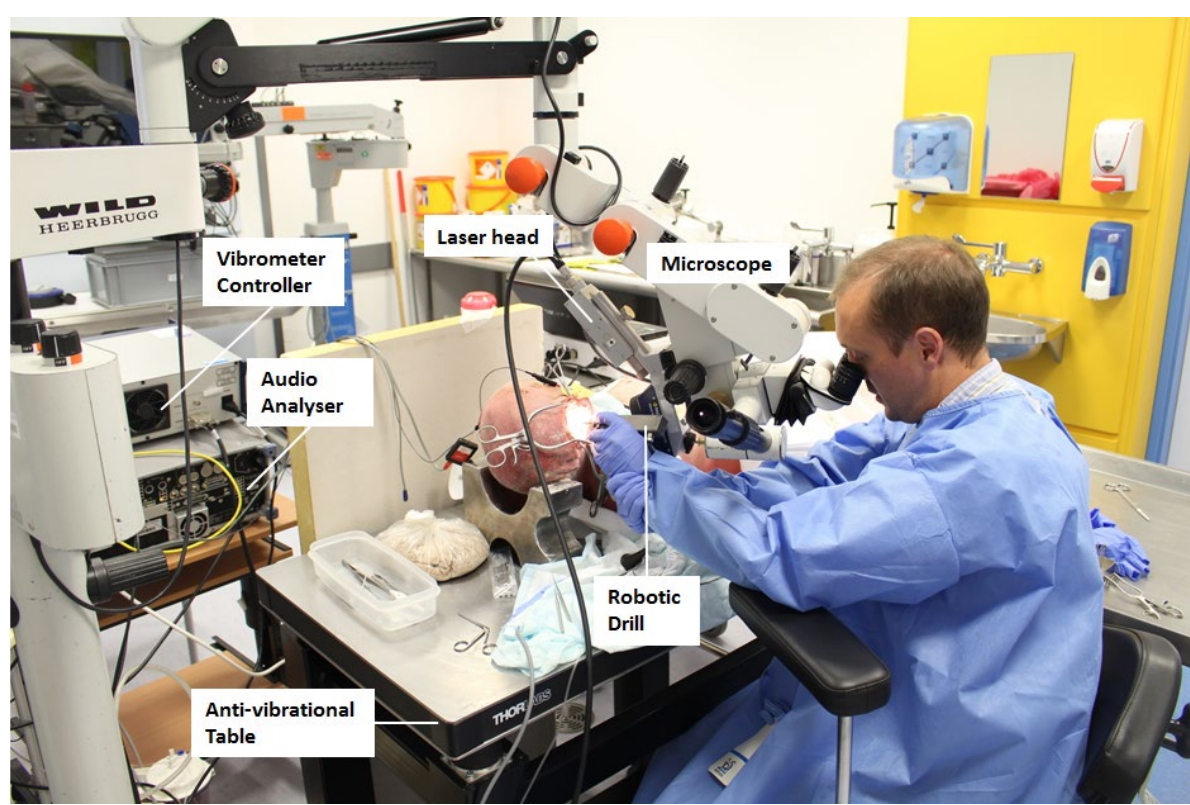

Figure 3 RW vibration measurement using LDV while the surgeon was performing cochleostomy drilling

Figure 3 is a comprehensive view of the laboratory setup of the measurement of RW response to cochleostomy drilling on human cadaver heads. The robotic drill was in use here. As illustrated in the figure, the surgeon's drilling arm was supported by the armrest of a surgery stool, which was ensured to bear no contact with the antivibrational table. This removes the direct transmission of the energy from hand and arm movement to the workbench, i.e. the anti-vibrational table where the cadaver head was laid. The surgeon's drilling hand was aided by his other hand to ease the maintenance of a consistent posture throughout the whole drilling session. The supporting arm was retained from touching the workbench for the same reason. All drilling processes were performed under the microscope, with the laser focused through the microscope on the retroreflective tape at the centre of RW. Care was made to ensure the laser beam remained on the retroreflective tape and that the beam was not interrupted by the surgeon's hand or instruments. Axial force exerted throughout the robotic drilling process was monitored and kept constant at approximately $1 \mathrm{~N}$ - the surgeon was able to correct the force applied according to a real-time indication signal. During the conventional drilling measurements, standard cochleostomy drilling surgical procedure and approach was followed and no attempt was especially made to apply constant pressure or remain contact. No irrigation was used in either drilling case, as this would interrupt the vibrometry signal.

\section{RESULTS}

\section{A. Cochleostomies and data processing}

Two complete cochleostomies were created. 1 was performed using robotic drill, while 2 with standard otologic drill. The milling, lifting and pushing motion during the conventional drilling procedure can make the opening slightly enlarged and not perfectly circular as manifested in Cochleostomy 2.

The round window velocity during drilling was measured by the laser vibrometer. A retroreflective tape was applied at the visually-estimated centre of the round window to aid the reflection of laser light. Sampling rate was set to $48 \mathrm{kHz}$ to cover the whole hearing frequency range of interest. Due to limited on-chip memory of the 
analyser, only a period of $10 \mathrm{~s}$ of data is achievable at every saving. To obtain a recording of the whole cochleostomy session, multiple continuously-taken 10-second recordings were attached in sequence in MATLAB. This recording of the full drilling session in the time domain was then processed through a set of algorithms to remove the unwanted off-target oscillation signals due to the unstable focus of the laser light. The 'off-target' events are typical to laser vibrometer measurement on a non-rigid moist biological membrane surface, and are artefacts introduced by the measurement procedure rather than the medical procedure under investigation. The limited size of the retroreflective tape $\left(<1 \mathrm{~mm}^{2}\right)$, in consideration of minimising mass load on the membrane, makes it more difficult to maintain laser reflection. The multiple 10-second recordings are attached in a sequence to form a raw data trace. The signal-to-noise ratio (SNR) is calculated by comparing signal power to the power of the ambient noise captured before drilling.

\section{B. Frequency Spectrum of Round Window Velocity}

The frequency spectrum of the drilling signal is obtained from the post-processing time series data, via fast Fourier transform (FFT). The frequency spectra for Cochlea A, B, C are plotted in Figure 4. Also plotted are the traces indicating the equivalent round window velocity if $100 \mathrm{~dB}$ and $85 \mathrm{~dB}$ sound is introduced into the ear, calculated from the METF described in Section 2B. The two traces are introduced because they denote critical thresholds for hearing protection. According to the National Institute for Occupational Safety and Health, long or repeated exposure to sounds at or above $85 \mathrm{~dB}$ can cause hearing loss. As a guide, the maximum time recommended that a healthy individual can be exposed to $100 \mathrm{~dB}$ sound is limited to $15 \mathrm{~min}$. Threshold time is halved for every $3 \mathrm{~dB}$ increase. Above all, the reference traces provide a snapshot of the level of disturbance in the context of hearing.

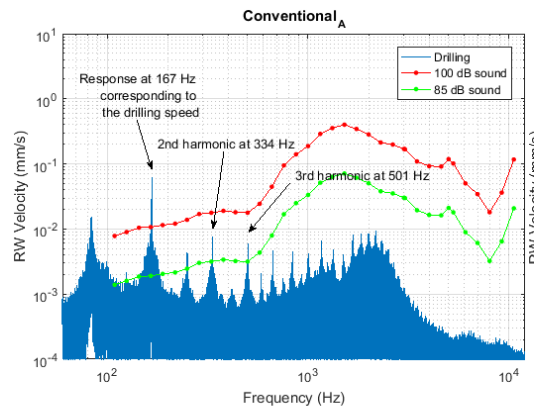

Robotic $_{\text {A }}$

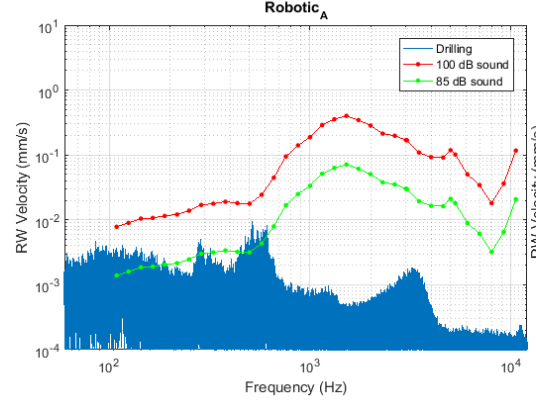

Cochlea A

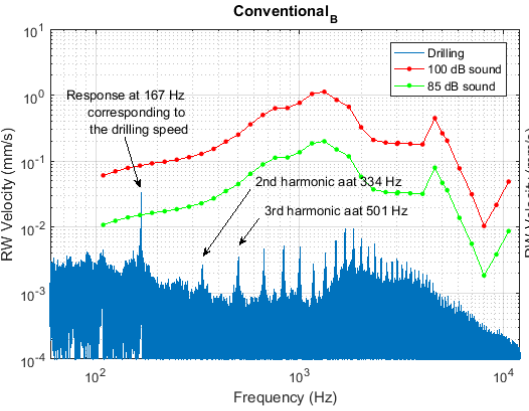

Robotic $_{\text {B }}$

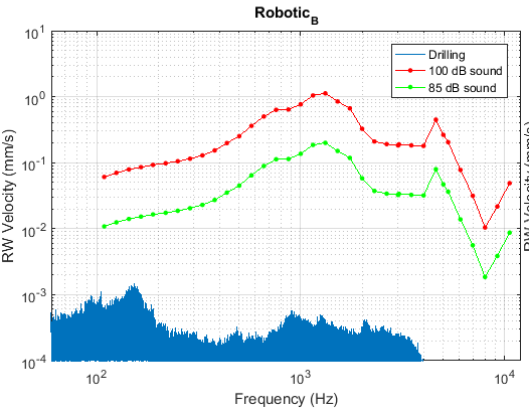

Cochlea B

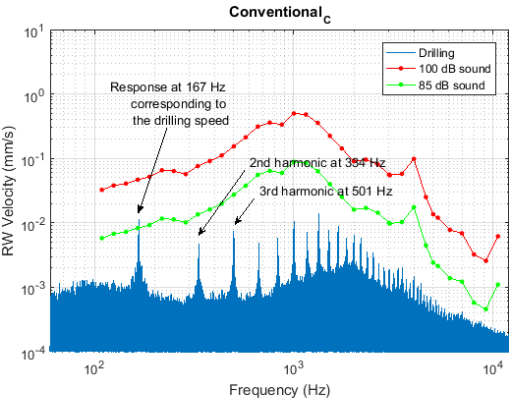

Robotic $_{\mathrm{c}}$

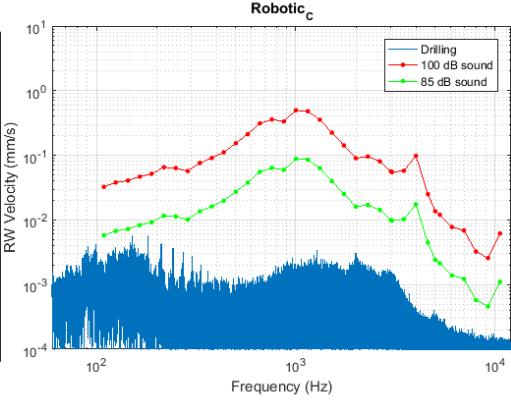

Cochlea C

Figure 4. Frequency spectrum of the drilling disturbance signal during the whole cochleostomy procedure. Top: conventional. Bottom: robotic.

\section{Formation Instantaneous Total Sound Pressure Level}

It is also possible to obtain the instantaneous equivalent sound pressure level from the data collected on cadaver heads, by accounting energy of all frequency components within the frequency range of interest. According to IEC standard [31], the sound pressure level is defined as:

$$
S P L=20 \cdot \log _{10} \frac{p}{p_{0}}
$$

For any particular time, the total sound pressure level can be calculated by substituting $p$ in the equation with the summation of the squares of the equivalent sound pressure at all frequencies [32]. Since the sound energy is proportional to the square of the sound pressure [33], this is equivalent to taking each frequency component as an independent source of energy and calculated the total impact of energy in units of dB SPL. 
Using the short-time Fourier transform and the METF-RW curve after interpolation, the time-resolved equivalent sound pressure levels can be determined. Instead of peak amplitude obtained straight from Fourier transform, root-mean-square amplitude of the sinusoidal component at each specific frequency is used here, to properly reflect the corresponding energy content $[34,35]$. The equivalent total sound pressure level is plotted against time in Figure 5. To facilitate the direct comparison between results that are of different recording lengths, the time is normalised by total cochleostomy time of each particular measurement. Accordingly, the calculation of sound pressure level is done in sliding sections of $1 \%$ of drilling time. The pain threshold of $120-140 \mathrm{~dB}$ SPL [36] is denoted by the red-shaded area in the figure. Frequency weighting is considered however not reported here since the threshold referred to (120-140 dB SPL) is an unweighted value.

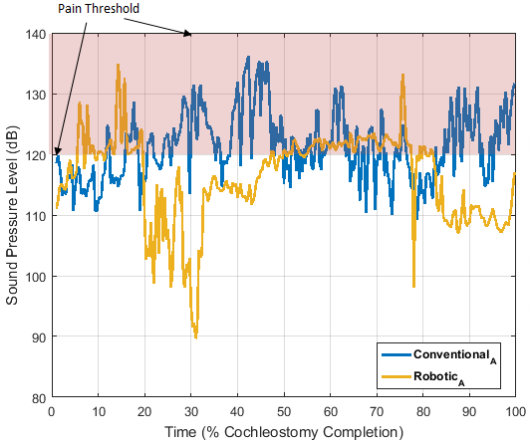

Cochlea A

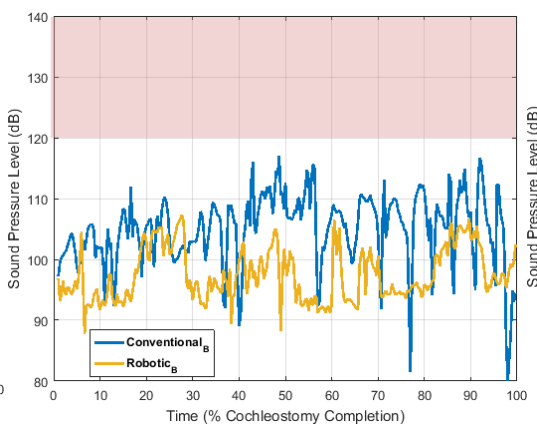

Cochlea B

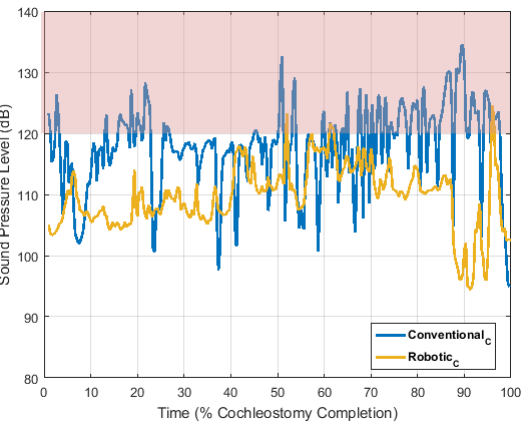

Cochlea C

Figure 5. Sound Pressure Level plotted against time which is normalised by total recorded cochleostomy drilling time. Shaded in red is the pain threshold of 120-140 dB SPL.

\section{DISCUSSION}

A direct comparison between conventional and robotic drilling on Cochlea A, B and C, is presented in Figure 6(a). Each bar value is the Root Mean Square (RMS) velocity of round window vibration over the whole procedure of cochleostomy drilling. In all three cases, robotic drilling delivers an RMS velocity that is approximately $1 / 3$ of that of conventional drilling. The peak amplitudes of equivalent sound pressure level are summarised in the Figure 6(b). A comparison between conventional and robotic, in respect of the peak amplitude of the frequency-specific equivalent sound pressure level is presented on top of the table. On all three cochlea specimen robotic drilling delivers a decrease in peak equivalent sound pressure level compared to conventional, ranging from $6 \%$ on Cochlea C to $29 \%$ on Cochlea B.
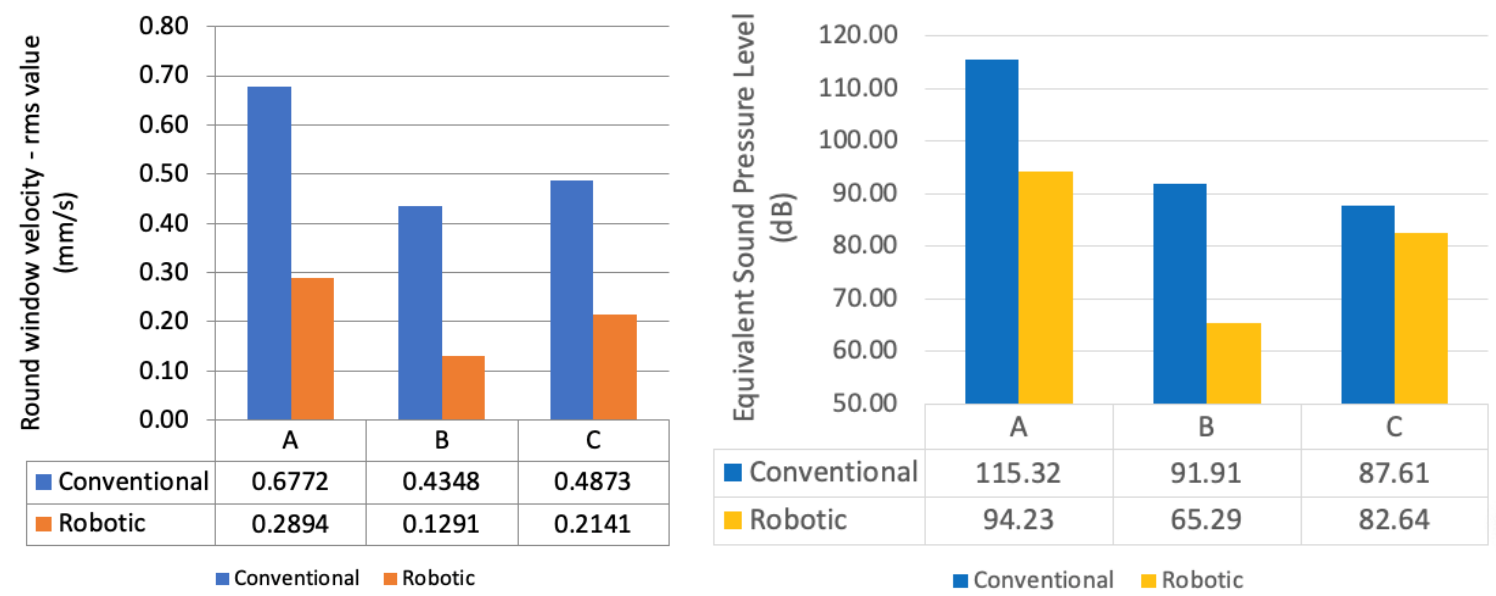

Figure 6. (a) Root mean square for round window velocity averaged over the whole cochleostomy procedure

(b) The peak amplitude of the induced mechanical disturbance in equivalent sound pressure level

From the results shown in Figure 5, the level of disturbance induced by robotic drilling is consistently lower, and 
amplitude during conventional drilling can be $20 \mathrm{~dB}$ SPL larger than that during robotic drilling, as per the trace of drilling-evoked equivalent sound pressure level over time. There is no obvious surge of disturbance level at the end of cochleostomy drilling which has been reported by other groups $[5,28]$. This can be most likely attributed to the discontinuing of both drilling and recording immediately upon completion of the fenestration. When drilling by robot, the running drill burr would have negligible or no direct contact with the exposed endosteum. Minimising trauma produced by physical disturbances is often the expressed goal in improving ear surgery as, in contrast, the resulting trauma is not readily understood or quantified. It is reasonably assumed that a reduction in disturbance in duration and amplitude will lead to reduced trauma. In cochleostomy drilling, the tissue guided robotic approach offers clear advantage.

\section{CONCLUSION}

Tissue guided robotic drilling offers advantage in minimising induced intracochlear disturbances over conventional surgical practice using conventional drills. The results have shown a consistently lower level of disturbance in cochlea both in the time domain and across the hearing frequency range. It is therefore reasonable to conclude that robotic drilling offers the possibility of reducing acoustic trauma in cochlea that currently places the residual hearing of patients at risk.

A hand-held robotic drill able to discriminate and respond to varying tissue types and conditions was compared with a conventional surgical drilling technique by an experienced otologist in cochlear implantation. In all three cases- there was a typical reduction of $20 \mathrm{~dB}$ when robotic drilling that is reflected by a reduction in RMS velocity at the round window to approximately $1 / 3$ of that of conventional drilling. The improvements are reinforced through the robotic approach by avoiding both intermittent removal and reconducting of the cutter, and by avoiding interaction with the endosteum with a running burr. These critical effects are unavoidable when controlled by a human operator seeking feedback on the state of the process, unable to stop the process on reaching the endosteum, and while compensating for induced tissue response to applied drilling force.

Although based on three cadaver specimens, the early results presented are promising. This indicates that the robotic approach has clear advantage in lowering disturbance to hearing organ. To consolidate and further establish the trend observed in this study, data from additional specimen is needed. This will diminish the likely effects imposed by variance in specimen condition, such as specimen freshness, anatomy, age, and gender.

\section{REFERENCES}

1. Miranda, P.C., Sampaio, A.L.L., Lopes, R.A.F., Ramos Venosa, A. and Oliveira, C.A.C.P.D., 2014. Hearing preservation in cochlear implant surgery. International journal of otolaryngology, 2014.

2. Royal National Throat Nose and Ear Hospital: Cochleaer implants for adults. (2014). University College London Hospitals NHS Foundation Trust

3. Cipolla, M. J., Iyer, P., Dome, C., Welling, D. B., \& Bush, M. L. (2012). Modification and comparison of minimally invasive cochleostomy techniques: A pilot study. The Laryngoscope, 122(5), 1142-7.

4. The American Hearing Research Foundation. (2008). Noise induced hearing loss. Retrieved January 1, 2016,

5. Pau, H. W., Just, T., Bornitz, M., Lasurashvilli, N., \& Zahnert, T. (2007). Noise exposure of the inner ear during drilling a cochleostomy for cochlear implantation. The Laryngoscope.

6. Yin, X., Strömberg, A.-K., \& Duan, M. (2011). Evaluation of the noise generated by otological electrical drills and suction during cadaver surgery. Acta Oto-Laryngologica, 131(11), 1132-1135.

7. Taylor, R.P., Du, X., Proops, D.W., Reid, A.P., Coulson, C., Brett, P.N.: A sensory-guided surgical microdrill. Proceedings of the Institution of Mechanical Engineers, Part C: Journal of Mechanical Engineering Science. 224(7): p. 1531-1537.

8. James C, Albegger K, Battmer R, Burdo S, Deggouj N, Deguine O, et al. "Preservation of residual hearing with cochlear implantation: How and why." Acta Oto-Laryngologica. 2005 5; 125(5):481-491.

9. Zou J, Bretlau P, Pyykkö I, Starck J, and Toppila E. "Sensorineural hearing loss after vibration: an animal model for evaluating prevention and treatment of inner ear hearing loss." Acta Otolaryngol. 2001 Jan; 121(2):143-148.

10. C. J. Coulson, M. Zoka Assadi, R. P. Taylor, X. Du, P. N. Brett, A. P. Reid, and D. W. Proops. "A smart micro-drill for cochleostomy formation: A comparison of cochlear disturbances with manual drilling and a human trial." Cochlear implants international 14, no. 2 (2013): 98-106.

11. Lanfranco, A.R., Castellanos, A.E., Desai, J.P. and Meyers, W.C., 2004. Robotic surgery: a current perspective. Annals of surgery, 239(1), p.14.

12. Guthart GS, Salisbury JK. "The Intuitive telesurgery system: overview and application." In IEEE International Conference on Robotics and Automation (ICRA ‘00), vol. 1, 2000: 618-621. 
13. Jakopec M, Rodriguez y Baena F, Harris SJ, et al. "The hands-on orthopaedic robot 'acrobot': early clinical trials of total knee replacement surgery." IEEE Trans Robotics Autom 2003; 19(5): 902-911.

14. Lonner JH, John TK, Conditt MA. "Robotic arm-assisted UKA improves tibial component alignment: a pilot study." Clin Orthop Relat Res 2010; 468(1): 141-146.

15. Hu JC, Gu X, Lipsitz SR, et al. Comparative effectiveness of minimally invasive vs open radical prostatectomy. JAMA. 2009;302(14):1557-64.

16. Ramsay C, Pickard R, Robertson C, et al. Systematic review and economic modelling of the relative clinical benefit and cost-effectiveness of laparoscopic surgery and robotic surgery for removal of the prostate in men with localised prostate cancer. Health Technol Assess. 2012;16(41):1-313.

17. Marco Caversaccio, Kate Gavaghan, Wilhelm Wimmer, Tom Williamson, Juan Anso, Georgios Mantokoudis, Nicolas Gerber et al. "Robotic cochlear implantation: surgical procedure and first clinical experience." Acta oto-laryngologica 137, no. 4 (2017): 447-454.

18. Omid Majdani, Thomas S. Rau, Stephan Baron, Hubertus Eilers, Claas Baier, Bodo Heimann, Tobias Ortmaier, Sönke Bartling, Thomas Lenarz, and Martin Leinung. "A robot-guided minimally invasive approach for cochlear implant surgery: preliminary results of a temporal bone study." International journal of computer assisted radiology and surgery 4, no. 5 (2009): 475-486.

19. Yann Nguyen, Mathieu Miroir, Jean-François Vellin, Stéphane Mazalaigue, Jean-Loup Bensimon, Daniele Bernardeschi, Evelyne Ferrary, Olivier Sterkers, and Alexis Bozorg Grayeli. "Minimally invasive computerassisted approach for cochlear implantation: a human temporal bone study." Surgical innovation 18, no. 3 (2011): 259-267.

20. Laskaris J, Regan K. Soft Tissue Robotics-The Next Generation. md buyline. 2014 Jun;7:1-3.

21. Jess H. Lonner, Glenn J. Kerr, "Robotically Assisted Unicompartmental Knee Arthroplasty." Operative Techniques in Orthopaedics, Volume 22, Issue 4, December 2012, Pages 182-188

22. A. Jaramaz, C. Nikou, and A. Simone, "NAVIOPFS FOR UNICONDYLAR KNEE REPLACEMENT: EARLY CADAVER VALIDATION" Bone Joint J 2013 95-B:(SUPP 28) 73.

23. Schuller, B.; Rigoll, G.; Can, S.; Feussner, H., "Emotion sensitive speech control for human-robot interaction in minimal invasive surgery," Robot and Human Interactive Communication, 2008. RO-MAN 2008. The 17th IEEE International Symposium on, vol., no., pp.453,458, 1-3 Aug. 2008

24. Nelson, C. A., Zhang, X., Shah, B. C., Goede, M. R., \& Oleynikov, D. (2010). Multipurpose surgical robot as a laparoscope assistant. Surgical endoscopy, 24(7), 1528-1532.

25. Payne CJ, Yang GZ. Hand-held medical robots. Annals of biomedical engineering. 2014 Aug 1;42(8):1594605 .

26. Yann Nguyen, Mathieu Miroir, Jean-François Vellin, Stéphane Mazalaigue, Jean-Loup Bensimon, Daniele Bernardeschi, Evelyne Ferrary, Olivier Sterkers, and Alexis Bozorg Grayeli. "Minimally invasive computerassisted approach for cochlear implantation: a human temporal bone study." Surgical innovation 18, no. 3 (2011): 259-267.

27. Du X, Assadi MZ, Jowitt F, Brett PN, Henshaw S, Dalton J, Proops DW, Coulson CJ, Reid AP. Robustness analysis of a smart surgical drill for cochleostomy. The International Journal of Medical Robotics and Computer Assisted Surgery. 2013 Mar 1; 9(1):119-26.

28. Eze N, Jiang D, Fitzgerald O'Connor A. Inner ear energy exposure while drilling a cochleostomy. Acta otolaryngologica. 2014 Nov 1;134(11):1109-13.

29. Rosowski JJ, Chien W, Ravicz ME, Merchant SN. Testing a method for quantifying the output of implantable middle ear hearing devices. Audiology and Neurotology. 2007;12(4):265-76.

30. Yu H, Tong B, Zhang Q, Zhu W, Duan M. Drill-induced noise level during cochleostomy. Acta otolaryngologica. 2014 Sep 1;134(9):943-6.

31. International Electrotechnical Commission. (2002). Letter symbols to be used in electrical technology - Part 3: Logarithmic and related quantities, and their units. IEC 60027-3 Ed. 3.0.

32. Guyer, P. (2009). Engineering SoundBite: Fundamentals of Acoustics. Guyer Partners.

33. Schnupp, J., Nelken, I., \& King, A. (2011). Auditory Neuroscience: Making Sense of Sound. MIT Press.

34. González-Prida, V. (2015). Promoting Sustainable Practices through Energy Engineering and Asset Management. IGI Global.

35. Scheffer, C., \& Girdhar, P. (2004). Practical Machinery Vibration Analysis and Predictive Maintenance. Elsevier Science.

36. Young ED, Fernandez C, Goldberg JM. Responses of squirrel monkey vestibular neurons to audio-frequency sound and head vibration. Acta oto-laryngologica. 1977 Jan 1;84(1-6):352-60. 